\title{
BMJ Open The heart \& mind trial: intervention with cognitive-behavioural therapy in patients with cardiac disease and anxiety: randomised controlled trial protocol
}

\author{
Selina Kikkenborg Berg (i) , ${ }^{1,2,3}$ Margrethe Herning, ${ }^{4}$ Inge Schjødt, ${ }^{5}$ \\ Charlotte Brun Thorup, ${ }^{6}$ Carsten Juul, ${ }^{7}$ Jesper Hastrup Svendsen, ${ }^{1,2}$ \\ Martin Balslev Jorgensen, ${ }^{2,8}$ Signe Stelling Risom, ${ }^{2,4,9}$ Signe Westh Christensen, ${ }^{1}$ \\ Lau Thygesen, ${ }^{3}$ Trine Bernholdt Rasmussen ${ }^{2,4}$
}

To cite: Berg SK, Herning M, Schjødt l, et al. The heart \& mind trial: intervention with cognitive-behavioural therapy in patients with cardiac disease and anxiety: randomised controlled trial protocol. BMJ Open 2021;11:e057085. doi:10.1136/ bmjopen-2021-057085

- Prepublication history for this paper is available online. To view these files, please visit the journal online (http://dx.doi. org/10.1136/bmjopen-2021057085).

Received 09 September 2021 Accepted 28 0ctober 2021

Check for updates

(c) Author(s) (or their employer(s)) 2021. Re-use permitted under CC BY-NC. No commercial re-use. See rights and permissions. Published by BMJ.

For numbered affiliations see end of article.

Correspondence to Professor Selina Kikkenborg Berg; selina@rh.dk

\section{ABSTRACT}

Introduction Patients with cardiac disease often experience anxiety (prevalence about 20\%-25\%) and have a doubled mortality risk when suffering from anxiety compared with patients without anxiety. This calls for interventions aiming to reduce anxiety.

Methods and analysis The Heart \& Mind Trial consists of three parts: (1) screening of all hospitalised and outpatient cardiac patients with arrhythmia, heart failure or ischaemic heart disease at four university hospitals in Denmark using the Hospital Anxiety and Depression ScaleAnxiety subscale (HADS-A); Patients scoring $\geq 8$ is invited to participate; (2) Assessment of the type of anxiety by Structured Clinical Interview for Diagnostic and Statistical Manual of Mental Disorders and (3) Randomised clinical superiority trial with blinded outcome assessment, with 1:1 randomisation to cognitive-behavioural therapy (CBT) performed by a CBT-trained cardiac nurse plus usual care or, usual care alone. The primary outcome is anxiety measured with HADS-A at 5 months. Secondary outcomes include anxiety symptoms measured with Becks Anxiety Inventory and heart rate variability. Exploratory outcomes measured at 12 months include blood cortisol (stress response), blood $\mathrm{C}$ reactive protein (stress response), health-related quality of life, readmission, mortality and attributable direct costs. A total of 336 patients will be included. The primary analyses are based on the intentionto-treat principle. For the primary outcome, we will use a linear regression model. For the long-term outcomes, mixed regression models will be used including repeated measurements.

Ethics and dissemination The trial is performed in accordance with the Declaration of Helsinki. All patients must give informed consent prior to participation and the trial is initiated after approval by the Danish Data Protection Agency (P-2020-894) and the National Committee on Health Research Ethics (H-20066739). Positive, neutral and negative results of the trial will be published.

Trial registration number NCT04582734.

\section{Strengths and limitations of this study}

- This is the first study to screen and diagnose anxiety in patients with cardiac disease and, to test a cognitive-behavioural therapy (CBT) intervention aimed at that specific type of anxiety.

- The interventional staff are cardiac nurses with CBT training, making this intervention a 'real life set-up' and easy to implement if results are positive.

- The trial does not investigate health anxiety which could occur.

- CBT may not be the most appropriate therapy for all the identified disorders; however, an anxiety disorder-specific approach is used.

- The trial has a patient-reported outcome measure, Hospital Anxiety and Depression Scale, as primary outcome but also include objective outcome measures such as heart rate variability and cortisol.

\section{INTRODUCTION}

Background and rationale

Patients with cardiac disease have a higher mortality risk when suffering from anxiety compared with patients without anxiety (when adjusted for age, sex, marital status and comorbidity). This was established in our research from $2018^{1}$ as well as found in other studies. ${ }^{2-6}$ It is therefore a natural next step to establish anxiety screening of patients to start an intervention aiming to reduce anxiety levels. Anxiety can be defined as a diffuse state "characterised by an unpleasant affective experience marked by a significant degree of apprehensiveness about the potential appearance of future aversive or harmful events. ${ }^{7} \mathrm{~A}$ formal nomenclature categorises the psychopathology anxiety disorders. ${ }^{8}$ Anxiety can be caused by the experience of living with 
an unpredictable disease and the risk of sudden cardiac death causing a significant negative influence on the individuals' quality of life. Many patients develop avoidance and safety behaviours that involve avoidance of physical activity and objects or places that they fear or that activate anxious feelings. This avoidance and safety behaviour may lead to social isolation and a situation characterised as a vicious circle with elevated anxiety levels and as a result, a further increased risk of death. ${ }^{9}$ Health anxiety may also be present as patients may fear suffering serious disease when in fact they don't. ${ }^{10}$ Studies indicate that rehabilitation initiatives, such as physical activity and cognitivebehavioural therapy (CBT) can reduce anxiety levels in cardiac patients. ${ }^{10-12}$

About $20 \%-25 \%$ of all cardiac patients experience symptoms of anxiety. ${ }^{13}$ Small differences exist between cardiac diagnostic groups. ${ }^{13}$ Data show that anxiety is the condition that causes the highest amount of lost work hours to society. ${ }^{14}$ Our research from 2018 showed that symptoms of anxiety predicted mortality (HR: 1.92, 95\% CI 1.52 to 2.42) in patients with cardiac disease across diagnostic groups. ${ }^{1}$ To take action to reduce the negative mental impact and the risk of premature death, it is important to screen cardiac patients for anxiety and intervene if psychopathological anxiety is detected. Both physiological and behavioural processes of anxiety may worsen undesired health outcomes. ${ }^{15}$ Suggested models of the relationship between psychology and heart disease underpin the role of the autonomic nervous system. Psychological factors stimulate the autonomic nervous system, which triggers the production of catecholamines, increase blood pressure, decrease plasma volume, promote vasoconstriction in coronary arteries, increase cardiac oxygen demand, increase platelet activity, as well as activation of coagulation and inflammation. These responses contribute to thrombogenesis, arrhythmogenesis, altered heart rate variability (HRV), increased myocardial oxygen demand, myocardial ischaemia and impaired ventricular function. ${ }^{16}$ Individuals with high anxiety (compared with non-anxious individuals) have unhealthier eating habits, ${ }^{17-19}$ smoke more,${ }^{17-20}$ consume more drugs and/or alcohol, ${ }^{17} 19$ are less compliant to treatment, ${ }^{21}$ have poor sleep quality ${ }^{1719}$ and are less physically active. ${ }^{1719}$ These are high-risk behaviours associated with increased incidence and progression of cardiac disease. ${ }^{22}$

A Cochrane review from 2017 reviewed psychological interventions for patients with coronary heart disease (CHD) ${ }^{12}$ The evidence suffers from being based on small trials evaluating multifactorial interventions on mixed populations of patients with and without psychological disorders. The review concludes that beneficial effects of psychological interventions are found for cardiac mortality, anxiety and depression, however, final conclusions are uncertain due to the low quality of the studies. ${ }^{12}$ Therefore, the authors advocate the need for large scale trials testing a specific psychological intervention (not multifactorial) for patients with CHD with or without psychopathologies. That means treatment or prevention.
There is no recommendation about treating anxiety differently by cardiac diagnosis however evidence is sparse in some diagnostic groups, for example, atrial fibrillation. ${ }^{11}$ Differences in type of anxiety around different cardiac diagnoses are dealt with in the same way as every other person with anxiety disorder have a different life story that may influence the onset and type of anxiety.

In deciding which specific intervention to test in the trial, the literature was searched. One review supports CBT as a first-line treatment for anxiety and depression in patients with cardiac disease as recommended by the National Institute for Health and Clinical Excellence. ${ }^{23} 24$ To maximise effects, face-to-face sessions should be prioritised as should the duration of CBT (four sessions or more). ${ }^{25}$ Internet-based interventions lack effect, often due to low adherence and high drop-out rates. ${ }^{26}$ Medical nurses with no psychological experience, after having received CBT training and supervision, provided significantly better patient outcomes than psychologists and other therapists. ${ }^{27}$ All these considerations and recommendations are included in the present interventional design of the Heart \& Mind Trial and include specific longer-lasting individual face-to-face CBT intervention, tested on a large group of cardiac patients with psychopathology anxiety $\geq 8$ Hospital Anxiety and Depression Scale (HADS), which is expected to be $25 \%$ of the total population. This means to investigate the type of anxiety and treat that specific type of anxiety the patients suffer from, which is also a part of the present intervention.

\section{Objectives}

The aim of the Heart \& Mind Trial is (1) to determine the type of anxiety in cardiac patients and (2) to investigate the effect of individual CBT intervention plus usual care to reduce anxiety compared with usual care alone in patients with cardiac disease and anxiety.

The hypothesis is that there will be a significant difference in anxiety scores (measured with HADS-Anxiety (HADS-A)) between intervention and usual care groups after intervention, in favour of the intervention group. Likewise, a difference is expected in depression, healthrelated quality of life (HeartQoL) and biological stress response outcomes.

\section{METHODS}

\section{Trial design}

The Heart \& Mind Trial is an investigator-initiated randomised clinical superiority trial with blinded outcome assessment, with 1:1 randomisation to CBT plus usual care or usual care alone.

Screening: Patients will be screened for anxiety using the HADS after being discharged from hospital. Screening will be administered with a delay of 8-12 weeks after discharge to allow for normal restitution. In addition, the patients will be screened at the outpatient clinic.

Patients with a HADS-A score $\geq 8$ will be interviewed using the Structured Clinical Interview for Diagnostic 


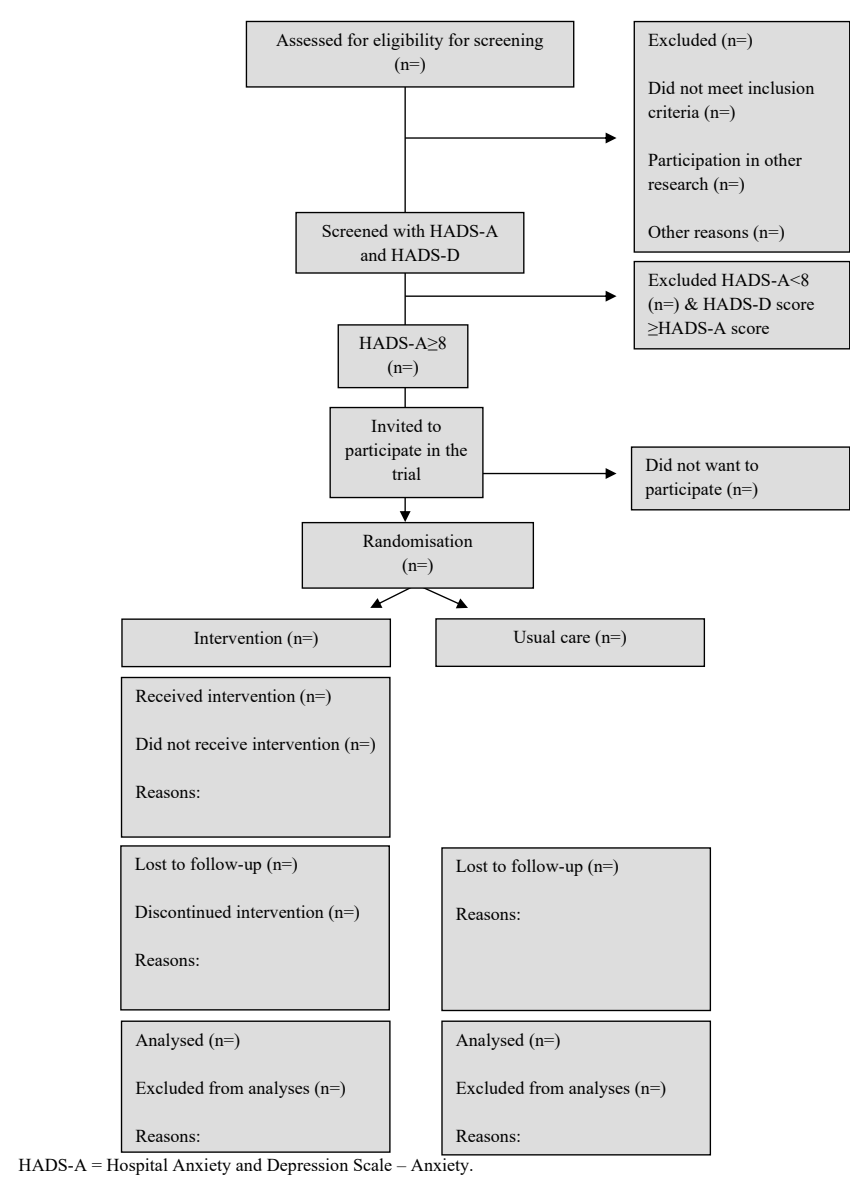

Figure 1 Flow chart. HADS-1, Hospital Anxiety and Depression Scale-Anxiety; HADS-D, HADS-Depression.

and Statistical Manuel of Mental (DSM) disorders (SCID) for DSM-IV manually delivered using the DSM-IV criteria in order to determine the type of anxiety patients suffer from. Thereafter participants will be randomised to intervention or usual care group following informed consent.

\section{Study setting}

Participants will be recruited from four Danish urban university hospital sites from three different regions of Denmark: Copenhagen University Hospital Rigshospitalet, Herlev and Gentofte University Hospital, Aarhus University Hospital and Aalborg University Hospital

\section{Eligibility criteria}

Patients are considered eligible for the trial if all of the following criteria are met before randomisation: $\geq 18$ years old, diagnosed with cardiac disease (arrhythmia, heart failure or ischaemic heart disease), treated one of the four inclusion sites, and score $\geq 8$ on the HADS-A. The HADS-A score must exceed the HADS-Depression (HADS-D) score. Participants must fulfil the criteria for anxiety or adjustment disorder as a result of the SCID-interview and must speak and understand Danish fluently and provide written informed consent to participate in the trial. The CONSORT flow chart is presented in figure 1.

\section{Experimental intervention}

The CBT intervention will aim at supporting patients to cope with their cardiac disease and treat the anxiety disorder. The CBT will be targeted on the specific type of anxiety based on the DSM-IV criteria. ${ }^{28}$ The therapy will focus on the patient's maladaptive thinking patterns, feelings and actions they engage in to cope with anxiety or the basic assumptions that cause these thoughts.

The methods of the therapeutic procedure are based on the learning theories and the fundamentals of CBT established by the psychiatrist Aaron T. Beck in the $1960 \mathrm{~s} .{ }^{29}$ For each type of anxiety (panic disorder, agoraphobia, social phobia, specific phobia, obsessive phobia, post-traumatic stress, generalised anxiety, anxiety due to a general medical condition, anxiety disorder not otherwise specified and adjustment disorder with anxiety) a specific CBT based protocol will be followed. The treatment of anxiety in cardiac patients will contain some of the following overall components:

1. Analysis of the problem: The patient's actual problems will be identified and related to their life story and events that influence this, for example, cardiac arrest. An investigation of the impact that assumptions and strategies established earlier in life have on the current problems and on the negative thoughts that are characteristic to anxiety.

2. Psychoeducation: Dissemination of information to the patient about anxiety, their cardiac disease and coping with everyday life. In relation to anxiety, most patients are helped by gaining insight into the sympathetic nervous system's role in relation to the development of it. In addition, the patients are informed about the association between negative automatic thoughts (catastrophic thoughts), bodily symptoms, feelings and actions, and how behaviour experiments/exposure may reduce anxiety in many cases.

3. Restructuring of negative automatic thoughts: Preparation of a 'thought journal' where the nurse and patient analyse problematic situations by identifying 'situation', 'physical symptoms', 'feelings', 'negative automatic thoughts' and 'behaviour'.

4. Planned behaviour experiments, consisting of systematic exposure to situations that trigger anxiety.

5. Homework, for example, registration of the relationship between thoughts, feelings and bodily sensations will be included in the sessions. These registrations form the foundation of content in the following therapy sessions, such as behaviour experiments, practical training or exercises in mastering. ${ }^{29}$

The intervention consists of weekly therapy sessions and is concluded when the patient has a HADS-A score $<8$ after two consecutive sessions. If the therapist assess that additional sessions are needed even after two sessions with a HADS-A score $<8$, it can be continued. After maximum 12 sessions in total regardless of score, the intervention is concluded. Intervention adherence is achieved by participating in the above number of consultations. 
There are no restrictions on concomitant care or medication during the trial period.

For the intervention to be carried out, the skills of the nurses must be upgraded. The skills upgrade involves a 10-day training course in CBT and SCID interview, followed by supervision by a psychologist, 6-15 two-hour sessions per employee spanning the intervention period. The process evaluation consists of monitoring dose delivered by nurses reporting number and duration of sessions, fidelity investigated by nurses monitoring which CBT worksheets is used in each session and if the patient has done the agreed homework.

\section{Usual care}

Both patient groups will receive usual care, which consists of medical therapy relevant to their cardiac disease and standard follow-up of their treatment according to current guidelines.

\section{Outcomes}

Demographic and clinical characteristics will be collected from patients and from patient records.

Ancillary questions: Information about all medication, blood pressure and pulse as well as patient-reported information about health-related behaviour including sleep quality, physical activity, alcohol consumption and smoking habits will be collected at baseline, 5 months and 12 months.

From patient records: Age, sex, type of heart disease and comorbidity, prior ventricular tachycardia (VT)/ ventricilar fribrillation (VF), New York Heart Association (NYHA) classification, ejection fraction, diabetes mellitus, time of cardiac diagnosis and all medication.

Data will be registered by trial staff when informed consent is obtained from the patient. Patient screening is done by patients filling out the self-reported HADS questionnaire in relation to their admission or appointment at one of the four sites. Included patients will be interviewed with the aim of determining the type of anxiety based on the SCID. ${ }^{28}$

\section{Primary outcome}

The primary outcome of the randomised controlled trial (RCT) is anxiety measured by HADS-A. HADS is a 14-item questionnaire that assesses anxiety and depression level in medically ill persons who are not admitted to psychiatric wards. The scale offers two scores, HADS-A and HADS-D, consisting of seven questions to assess anxiety and seven to assess depression. The respondent must indicate how they have been feeling in the past week. HADS is a validated tool with a Cronbach's $\alpha$ of 0.83 and 0.82 for the anxiety and depression subscales, respectively. Evidence of convergent validity and high internal consistency for both HADS outcomes was found in a large sample of Danish patients with cardiac disease.$^{30}$ Scores of $0-7$ for either subscale are regarded as normal, scores of 8 to 10 suggest the presence of a mood disorder, and scores of 11 and above suggest the probable presence of a mood disorder. ${ }^{31}$ HADS is measured at baseline, at every CBT session (intervention group only), at 5 months (primary outcome), and at 12 months (long-term explorative outcome).

\section{Secondary outcomes}

Becks Anxiety Inventory (BAI) is a self-reported measure of anxiety with a focus on somatic symptoms of anxiety. It was developed as a measure to discriminate between anxiety and depression. ${ }^{32}$ The 21-item questionnaire assesses symptoms such as nervousness, dizziness and fear of dying. For each item, the patient is asked to report how he or she has felt during the past week. The BAI score ranges from 0 to 63 and is interpreted as follows: $0-9$, normal or no anxiety; 10-18, mild to moderate anxiety; 19-29, moderate to severe anxiety and 30-63, severe anxiety. The BAI has been proven to be highly internally consistent with a Cronbach's $\alpha$ of $0.94 .{ }^{33} \mathrm{BAI}$ is measured at baseline, at 5 and 12 months.

HRV is measured in beats per minute with a portable device for continuous monitoring of various electrical variables of the cardiovascular system (Evo, Spacelab USA and Epatch, Biotelemetry). HRV, blood pressure and heart rate are all responsive to sympathetic activity, which can be increased by anxiety. HRV refers to the beat-to-beat variation in the $R R$ interval and is a marker of autonomic nervous system activity. ${ }^{34}$ Reduced HRV is a powerful and independent predictor of short and long-term mortality in cardiac patients. ${ }^{35}{ }^{36}$ Higher levels of anxiety are associated with reduced HRV. ${ }^{37}$ Holter recordings with $>100$ / hour premature ventricular contractions are excluded from the analyses as are patients that are paced more than $50 \%$ and those with cardiac resynchronisation therapy. Sinus rhythms with non-paced beats were used. HRV is measured at baseline and 5 months.

\section{Exploratory outcomes}

Cortisol is a stress marker and the hypothesis is that that there will be a difference between control and intervention groups in serum cortisol, with higher levels found in the control group. Patients are tested from 8:00-10:00 hours to minimise fluctuations. Measured at baseline and 5 months.

$\mathrm{C}$ reactive protein (CRP) is an inflammatory marker and the hypothesis is that that there will be a difference between control and intervention groups in serum CRP, with higher levels found in the control group. Measured at baseline and 5 months.

Cortisol and CRP will be collected through blood samples $(4-8 \mathrm{~mL})$ at baseline and 5 months when the patients are screened for anxiety (HADS-A) at the site. The samples will be destroyed after the analyses of cortisol and CRP.

HeartQoL is a disease-specific questionnaire that measures cardiac HeartQoL and was developed in patients with ischaemic heart disease. The questionnaire consists of 14 items and provides an overall global score and two subscales; a 10-item physical subscale and a 4-item 
emotional subscale, which are scored from 0 to $3 .^{38}$ The questionnaire asks patients to remember how their heart condition has bothered them in the past 4 weeks. ${ }^{38}$ It has proven to be a reliable instrument with a Cronbach's $\alpha$ between 0.80 and 0.91 for the global score and each subscale and to be responsive in patients with a wide spectrum of cardiac diagnoses. ${ }^{38-40}$ HeartQoL is measured at baseline, 5 months and 12 months.

Data on acute and planned admissions will be collected through The Danish National Patient Register 12 months after the end of the intervention. ${ }^{41}$
Data on mortality will be collected through the Civil Registration System 12 months after the end of the intervention. ${ }^{42}$

Information on individual-level costs will be collected for contacts with the hospital and the primary healthcare sector through the Danish National Health Service Register and the Danish National Patient Register. ${ }^{41} 43$

\section{Participant timeline}

The timeline is presented in figure 2.

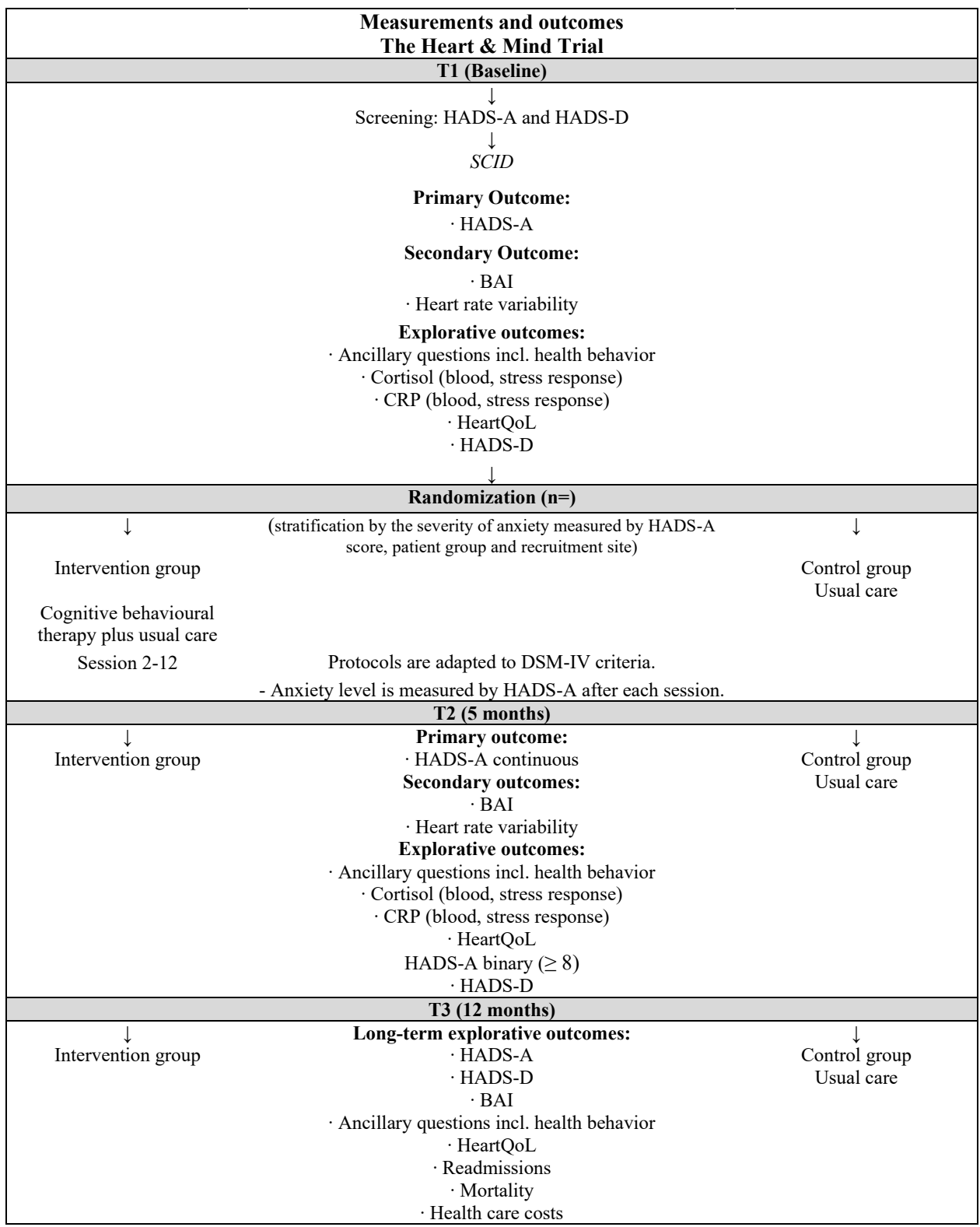

$\mathrm{BAI}=$ Becks Anxiety Inventory, $\mathrm{CRP}=\mathrm{C}$-reactive protein, $\mathrm{DSM}=$ Diagnostic and Statistical Manual of Mental Disorders, HADS $=$ Hospital Anxiety and Depression Scale, SCID = Structured Clinical Interview for DSM disorders.

Figure 2 Participant timeline. BAI, Becks Anxiety Inventory; CRP, C reactive protein; DSM-IV, Diagnostic and Statistical Manuel of Mental-IV; HADS-1, Hospital Anxiety and Depression Scale-Anxiety; HADS-D, HADS-Depression; HeartQoL, health-related quality of life; SCID, Structured Clinical Interview for DSM disorders. 


\section{Sample size and power estimations}

Sample size

It has previously been found that among cardiac patients the minimal clinically important difference on the HADS is 1.7 points. ${ }^{44}$ In a trial investigating the effect of CBT on patients with implantable cardioverter defibrillator (ICD) and anxiety, ${ }^{45}$ a difference between groups of four points was found; intervention group 4.95 (SD 3.30) versus usual care group 8.98 (SD 4.03), $\mathrm{p}<0.0001$, Cohen's d -0.86 . Therefore, an expected difference of 2 points seems reasonable. The SD is found to be 2.7 in a group of 3250 patients with heart disease and HADS-A above 8 (unpublished data from the DenHeart dataset).

With a power of $90 \%$ and a type 1 error set at 0.05 , a total of 39 patients should be entered into each group. If the risk of type I error is reduced to 0.01 and the expected minimal difference is still two points then 56 patients should be entered into each group. To allow for subanalyses on each of the main groups, arrhythmia, heart failure and ischaemic heart disease, we include a total of 336 patients (168 intervention patients and 168 control patients) equally distributed among the three main diagnostic groups to have sufficient statistical power.

\section{Power estimations for secondary outcomes}

BAI: In a previous study, the response within each subject group was normally distributed with an SD of ${ }^{46}$ If the true difference between the intervention and control group means is $5.2^{45}$ we will be able to reject the null hypothesis that the population means of the intervention and control groups are equal with a probability (power) $>0.999$.

HRV (SDNNi): In a previous study the response within each subject group was normally distributed with a SD of 17.3 (6). If the true difference in the intervention and control group means is 6.32 , we will be able to reject the null hypothesis that the population means of the intervention and control groups are equal with a probability (power) of 0.914 .

\section{Recruitment}

All patients 18 years or older with a cardiac disease (arrhythmia, heart failure or ischaemic heart disease), who are discharged from or have an outpatient appointment at one of the four inclusion sites during the trial inclusion period are invited to fill out the HADS screening for anxiety and interviewed by SCID to diagnose anxiety. In patients who have been admitted to the hospital, the screening will take place at least 8 weeks after an event or hospital discharge to allow for normal restitution. The screening will continue until the target sample size is achieved.

If a patient, after receiving both verbal and written information, decides to participate in the Heart \& Mind Trial, an informed consent form will be signed, and patients will be randomised to either: (1) a CBT intervention and usual care or (2) usual care alone. The intervention is performed by cardiac nurses with certified CBT training. The nurses are trained and supervised by a psychologist.

\section{Allocation}

Patients will be randomised 1:1 to the intervention group or to the control group. Randomisation will be conducted using the web-based tool Randomiser for Clinical Trials. The allocation sequence will be computer-generated with a block size of 4 , concealed from the investigators. The allocation will be conducted when the investigator calls a voice respondent who logs in to 'Randomiser for Clinical Trials 1.8.1' and selects relevant participant information (participant number and stratum) and assigns the participant to either intervention or control group by phone to the investigator. The strata are: severity of anxiety measured by HADS-A (8-10 or 11-21), recruitment site (four sites) and type of cardiac disease (arrhythmia, heart failure or ischaemic heart disease).

\section{Blinding}

Because of the conditions required for psychoeducational interventions, it is not possible to blind the intervention staff and patients. All baseline information and clinical interviews are collected and performed before randomisation. Physical tests, data collection, data management and administration will be done by blinded staff. Statistical analysis of outcomes and conclusions from these will be blinded as well. The results of the trial will be analysed by an independent statistician, and the results will be interpreted by the research group. The conclusion will be prepared in two versions, before the allocation code is broken, with the two arms alternately assumed as intervention (one that assumes that $\operatorname{arm} \mathrm{A}$ is the intervention group, and a second that assumes that arm B is the intervention group).

\section{Data collection}

The questionnaires for screening and collection of BAI and HeartQoL are self-administered and handed out to the patient at the hospital or sent to their home address. Measures of HRV, cortisol and CRP will be collected at one of the four sites at the first project consultation.

The SCID interviews are performed by intervention nurses. The first interviews are performed by two nurses together to allow for training and assure inter-rater concordance.

Trial patients are free to withdraw their informed consent at any time and be treated according to the department's standard procedures. Patients who leave the trial will be asked for permission to continue to collect data and to use already collected data. If the patient gives permission, data will be included in the final analyses.

\section{Data management}

Study data will be collected and managed using Research Electronic Data Capture (REDCap) hosted at Rigshospitalet, Copenhagen University Hospital. ${ }^{47}{ }^{48}$ REDCap is a secure, web-based software platform designed to support data capture for research studies, providing (1) an intuitive interface for validated data capture; (2) audit trails for tracking data manipulation and export procedures; 
(3) automated export procedures for seamless data downloads to common statistical packages and (4) procedures for data integration and interoperability with external sources. ${ }^{47} 48$

All completed questionnaires and informed consent forms signed by patients will be stored in locked filing cabinets in areas with limited access at the sites.

Individual patient data will be handled as normal data and records will be protected according to the Act on Processing of Personal Data and the Danish Healthcare Acts. Data will be stored in accordance with Danish Data Protection Agency rules. Data that is encoded with the individual patient code will be entered into the computerised REDCap database and transferred for analysis portal in encrypted mode. This system meets all criteria for the handling of patient data in accordance with the laws on the processing of personal data. The trial database will be preserved for 15 years and anonymised. After analysis, experimental data will be submitted to the Danish Data Archives.

\section{Statistical analysis}

The primary analyses will be performed according to the intention-to-treat principle. The primary outcome will be analysed by a linear regression model with adjustment for the stratification variables (HADS-A, recruitment site and cardiac disease). The results for the primary outcome will also be reported by mean values and t-tests. For the other continuous outcomes, linear regression models will be used, while binary outcomes will be analysed by a logistic regression model. For the long-term outcomes, mixed regression models (linear and logistic) will be used including repeated measurements. By using mixed models, we ensure that missing data does not create bias if they are missing at random. If the proportion of missing data of the outcomes at 5 months is larger than $20 \%$, missing data will be imputed using multiple imputations. In case of significant results in the primary and secondary outcomes, sensitivity analyses will be performed to estimate the potential effect of data missing by imputing missing values as the baseline value. Readmission and mortality will be analysed by Cox regression analyses. Cost data will be analysed by linear regression models. The clinical effect size is analysed by Cohen's d. The significance level is set at $5 \%$.

\section{Data monitoring}

Due to no harms expected ${ }^{45}$ and expected fast inclusion, a data monitoring committee is not established, and no interim analyses will be performed.

\section{Harms}

No risks are expected to occur during the trial. CBT is a safe non-invasive, non-pharmacological treatment. There is a potential beneficial effect of participation in CBT as anxiety levels may decrease. ${ }^{49}$ To avoid interference with normal restitution an 8-week time span after an event or hospital discharge must be upheld before screening for anxiety. Adverse events will be continuously monitored.

\section{Patient and public involvement statement}

A trial, testing the anxiety trial design on 88 patients with ICD at two sites (ClinicalTrials.gov (NCT02713360)) had been conducted with good feasibility and positive outcomes. Process evaluation will be carried out during the trial in order to explore the implementation, receipt and setting of the intervention. ${ }^{50}$ Interviews will be held with administrative management, nurses and patients in order to assess their opinions regarding the importance of the intervention (pretrial) and organisational barriers to implementation (trial setup), the employee's experiences delivering the intervention and the patients' experience of receiving it (end-trial).

\section{ETHICS AND DISSEMINATION \\ Ethics}

The trial is performed in accordance with the Declaration of Helsinki in its latest form. All patients must give informed consent to the investigators before participation. All trial patients are informed that all their personal information is confidential. The trial is initiated after approval by the Danish Data Protection Agency (P-2020894) and the National Committee on Health Research Ethics (H-20066739). The National Committee on Health Research Ethics will be asked for permission in case of protocol amendments. All investigators will be given full access to the final trial data set.

\section{Dissemination}

Positive, neutral and negative results of the trial will be published. The final manuscripts originating from the trial will be sent to a peer-reviewed international journal. Authorship will be allocated using the guidelines for authorship set out by the International Committee of Medical Journal Editors and will depend on the personal involvement of each author. The trial is expected to begin in April 2021 with the inclusion of the first patient. Inclusion will end when 336 patients are enrolled in the RCT, expected to be by the end of 2021. Data collection will end by end-2022. The trial may be delayed due to the COVID-19 pandemic.

\section{Author affiliations}

${ }^{1}$ The Heart Centre, Copenhagen University Hospital, Rigshospitalet, Copenhagen 0, 2100, Denmark

${ }^{2}$ Department of Clinical Medicine, Faculty of Health and Medical Sciences, University of Copenhagen, Copenhagen N, 2200, Denmark

${ }^{3}$ National Institute of Public Health, University of Southern Denmark, Copenhagen K, 1455, Denmark

${ }^{4}$ Department of Cardiology, Herlev and Gentofte University Hospital, Hellerup, 2900, Denmark

${ }^{5}$ Department of Cardiology, Aarhus University Hospital, Aarhus, 8200, Denmark ${ }^{6}$ Clinical Nursing Research Unit and Department of Cardiology, Aalborg University Hospital, Aalborg, 9000, Denmark

${ }^{7}$ Psychological consulting, Heypeople, Copenhagen K, 1260, Denmark

${ }^{8}$ Psychiatric Centre Copenhagen, Copenhagen University Hospital, Rigshospitalet, Copenhagen 0,2100, Denmark 
${ }^{9}$ University College Copenhagen, Institute of Nursing and Nutrition, Copenhagen N, 2200, Denmark

Contributors SKB conceived the idea of the study. SKB, MH, IS, CBT, CJ, JHS, MJ, SSR, SWC, LT and TBR planned and conducted the study design. LT and SKB developed the plan for the statistical analyses. All authors contributed to the reporting of the study protocol.

Funding The Heart \& Mind Trial will be conducted at the Heart Centre, Copenhagen University Hospital Rigshospitalet, at the Department of Cardiology, Herlev and Gentofte University Hospital, at the Department of Cardiology, Aarhus University Hospital and at the Department of Cardiology, Aalborg University Hospital. This work was supported by The Novo Nordisk Foundation, grant number (NNF180C0034016), The Capital Region of Denmark, grant number (A5972) and The Danish Nurses' Association's Nursing Research Fund (N/A). The trial will be funded by external funds for research in health sciences.

Competing interests None declared.

Patient and public involvement Patients and/or the public were involved in the design, or conduct, or reporting, or dissemination plans of this research. Refer to the Methods section for further details.

Patient consent for publication Not applicable.

Provenance and peer review Not commissioned; externally peer reviewed.

Open access This is an open access article distributed in accordance with the Creative Commons Attribution Non Commercial (CC BY-NC 4.0) license, which permits others to distribute, remix, adapt, build upon this work non-commercially, and license their derivative works on different terms, provided the original work is properly cited, appropriate credit is given, any changes made indicated, and the use is non-commercial. See: http://creativecommons.org/licenses/by-nc/4.0/.

\section{ORCID iD}

Selina Kikkenborg Berg http://orcid.org/0000-0002-9493-954X

\section{REFERENCES}

1 Berg SK, Thorup CB, Borregaard B, et al. Patient-reported outcomes are independent predictors of one-year mortality and cardiac events across cardiac diagnoses: findings from the national DenHeart survey. Eur J Prev Cardiol 2019;26:624-37.

2 Geulayov G, Novikov I, Dankner D, et al. Symptoms of depression and anxiety and 11-year all-cause mortality in men and women undergoing coronary artery bypass graft (CABG) surgery. $J$ Psychosom Res 2018;105:106-14.

3 de Jager TAJ, Dulfer K, Radhoe S, et al. Predictive value of depression and anxiety for long-term mortality: differences in outcome between acute coronary syndrome and stable angina pectoris. Int J Cardiol 2018;250:43-8.

4 van Dijk MR, Utens EMWJ, Dulfer K, et al. Depression and anxiety symptoms as predictors of mortality in $\mathrm{PCl}$ patients at 10 years of follow-up. Eur J Prev Cardiol 2016;23:552-8.

5 Watkins LL, Koch GG, Sherwood A, et al. Association of anxiety and depression with all-cause mortality in individuals with coronary heart disease. J Am Heart Assoc 2013;2:e000068.

6 Kikkenborg Berg S, Caspar Thygesen L, Hastrup Svendsen J, et al. Anxiety predicts mortality in ICD patients: results from the crosssectional national CopenHeartICD survey with register follow-up. Pacing Clin Electrophysiol 2014;37:1641-50.

7 DiTomasso RA, Gosch E. Anxiety disorders: a practitioner's guide to comparative treatment. New York: Springer, 2002.

8 The American Psychiatric Association. DSM-5 diagnostic and statistical manual of mental disorders. 5th edn. Washington DC: American Psychiatric Association, 2013: 947.

9 Dunbar SB, Dougherty CM, Sears SF, et al. Educational and psychological interventions to improve outcomes for recipients of implantable cardioverter defibrillators and their families: a scientific statement from the American heart association. Circulation 2012;126:2146-72.

10 Tyrer P, Cooper S, Salkovskis P, et al. Clinical and cost-effectiveness of cognitive behaviour therapy for health anxiety in medical patients: a multicentre randomised controlled trial. Lancet 2014;383:219-25.

11 Reavell J, Hopkinson M, Clarkesmith D, et al. Effectiveness of cognitive behavioral therapy for depression and anxiety in patients with cardiovascular disease: a systematic review and meta-analysis. Psychosom Med 2018;80:742-53.
12 Richards SH, Anderson L, Jenkinson CE. Psychological interventions for coronary heart disease. Cochrane Database Syst Rev 2017.

13 Berg SK, Rasmussen TB, Thrysoee L, et al. DenHeart: differences in physical and mental health across cardiac diagnoses at hospital discharge. J Psychosom Res 2017;94:1-9.

14 Sundhedsstyrelsen. Sygdomsbyrden I Danmark. Copenhagen: Sundhedsstyrelsen, 2015.

15 eds. Dejong MJ. Impact of anxiety on cardiac disease. In: Moser DK, Riegel B, eds. Caridac nursing: a companion to Braunwald's heart disease. St. Louis, Missouri: Saunders, 2008: 533-42.

16 Molinari E, Parati G, Compare A. Clinical psychology and heart disease. Milano: Springer Milan, 2006: 509.

17 Buselli EF, Stuart EM. Influence of psychosocial factors and biopsychosocial interventions on outcomes after myocardial infarction. J Cardiovasc Nurs 1999;13:60-72.

18 Hayward C. Psychiatric illness and cardiovascular disease risk. Epidemiol Rev 1995;17:129-38.

19 Sirois BC, Burg MM. Negative emotion and coronary heart disease. A review. Behav Modif 2003;27:83-102.

20 Kubzansky LD, Kawachi I, Weiss ST, et al. Anxiety and coronary heart disease: a synthesis of epidemiological, psychological, and experimental evidence. Ann Behav Med 1998;20:47-58.

21 Frasure-Smith $\mathrm{N}$, Lespérance $\mathrm{F}$, Talajic M. The impact of negative emotions on prognosis following myocardial infarction: is it more than depression? Health Psychol 1995;14:388-98.

22 Moser DK, Riegel B. Cardiac nursing: a companion to braunwald's heart disease. Missouri: Elsevier Saunders, 2008.

23 National Institute for Health and Clinical Excellence (NICE). Anxiety disorders quality standard. NICE, 2014.

24 National Institute for Health and Clinical Excellence (NICE). Depression in adults: treatment and management. NICE, 2017: 1-63.

25 Reavell J, Hopkinson M, Clarkesmith D. Effectiveness of cognitive behavioral therapy for depression and anxiety in patients with cardiovascular disease. Psychosom Med 2018;1.

26 Norlund F, Wallin E, Olsson EMG, et al. Internet-Based cognitive behavioral therapy for symptoms of depression and anxiety among patients with a recent myocardial infarction: the U-CARE heart randomized controlled trial. J Med Internet Res 2018;20:e88.

27 Tyrer H, Tyrer P, Lisseman-Stones Y. Therapist differences in a randomised trial of the outcome of cognitive behaviour therapy for health anxiety in medical patients. Int J Nurs Stud 2015;52:686-94.

28 Williams JBW. The structured clinical interview for DSM-III-R (SCID). Arch Gen Psychiatry 1992;49:630.

29 Arendt M, Rosenberg NK. Kognitiv terapi - Nyeste udvikling. Hans Reitzels Forlag, 2012.

30 Christensen AV, Dixon JK, Juel K, et al. Psychometric properties of the Danish Hospital anxiety and depression scale in patients with cardiac disease: results from the DenHeart survey. Health Qual Life Outcomes 2020;18:9.

31 Bjelland I, Dahl AA, Haug TT, et al. The validity of the hospital anxiety and depression scale. J Psychosom Res 2002;52:69-77.

32 Beck AT, Epstein N, Brown G, et al. An inventory for measuring clinical anxiety: psychometric properties. J Consult Clin Psychol 1988;56:893-7.

33 Fydrich T, Dowdall D, Chambless DL. Reliability and validity of the beck anxiety inventory. J Anxiety Disord 1992;6:55-61.

34 Heart rate variability: standards of measurement, physiological interpretation and clinical use. Task force of the European Society of cardiology and the North American Society of pacing and electrophysiology. Circulation 1996;93:1043-65.

35 Stein PK, Domitrovich PP, Huikuri HV, et al. Traditional and nonlinear heart rate variability are each independently associated with mortality after myocardial infarction. J Cardiovasc Electrophysiol 2005;16:13-20.

36 Aronson D, Mittleman MA, Burger AJ. Measures of heart period variability as predictors of mortality in hospitalized patients with decompensated congestive heart failure. Am J Cardiol 2004;93:59-63.

37 Carney RM, Blumenthal JA, Stein PK, et al. Depression, heart rate variability, and acute myocardial infarction. Circulation 2001;104:2024-8.

38 Oldridge N, Höfer S, McGee H, et al. The HeartQoL: Part I. development of a new core health-related quality of life questionnaire for patients with ischemic heart disease. Eur J Prev Cardiol 2014;21:90-7.

39 Oldridge N, Saner H, McGee HM, et al. The Euro Cardio-QoL project. An international study to develop a core heart disease health-related quality of life questionnaire, the HeartQoL. Eur J Cardiovasc Prev Rehabil 2005;12:87-94. 
40 Oldridge N, Höfer S, McGee H, et al. The HeartQoL: Part II. validation of a new core health-related quality of life questionnaire for patients with ischemic heart disease. Eur J Prev Cardiol 2014;21:98-106.

41 Lynge E, Sandegaard JL, Rebolj M. The Danish national patient register. Scand J Public Health 2011;39:30-3.

42 Pedersen CB. The Danish civil registration system. Scand J Public Health 2011;39:22-5.

43 Andersen JS, Olivarius NDF, Krasnik A. The Danish National health service register. Scand J Public Health 2011;39:34-7.

44 Lemay KR, Tulloch HE, Pipe AL. Establishing the minimal clinically important difference for the hospital anxiety and depression scale in patients with cardiovascular disease. $J$ Cardiopulm Rehabil Prev 2018;1.

45 Berg SK, Rasmussen TB, Herning M, et al. Cognitive behavioural therapy significantly reduces anxiety in patients with implanted cardioverter defibrillator compared with usual care: findings from the Screen-ICD randomised controlled trial. Eur J Prev Cardiol 2020;27:258-68.
$46 \mathrm{Kim}$ YW, Lee S-H, Choi TK, et al. Effectiveness of mindfulness-based cognitive therapy as an adjuvant to pharmacotherapy in patients with panic disorder or generalized anxiety disorder. Depress Anxiety 2009;26:601-6.

47 Harris PA, Taylor R, Thielke R, et al. Research electronic data capture (REDCap)--a metadata-driven methodology and workflow process for providing translational research informatics support. J Biomed Inform 2009;42:377-81.

48 Harris PA, Taylor R, Minor BL, et al. The REDCap Consortium: building an international community of software platform partners. $J$ Biomed Inform 2019;95:103208.

49 Maia ACCO, Braga AA, Soares-Filho G, et al. Efficacy of cognitive behavioral therapy in reducing psychiatric symptoms in patients with implantable cardioverter defibrillator: an integrative review. Braz $J$ Med Biol Res 2014:47:265-72.

50 Oakley A, Strange V, Bonell C, et al. Process evaluation in randomised controlled trials of complex interventions. $B M J$ 2006;332:413-6. 